\title{
100 International Units per Milliliter
}

National Cancer Institute

\section{Source}

National Cancer Institute. 100 International Units per Milliliter. NCI Thesaurus. Code C71185.

A unit of arbitrary substance concentration (biologic activity concentration) defined as the concentration of one hundred international units per one milliliter of system volume. 